\title{
Summary of Seven More Studies on Numerosity Abilities in an Ant, Four of Them Relating to Human Competence
}

\author{
Marie-Claire Cammaerts
}

Independent researcher, retired from the Department of Biology of Organisms, University of Brussels. Present and corresponding postal address: 27, Square du Castel Fleuri, 1170

Bruxelles, Belgium. Tel.: 32267349 69. E-mail: mccammaerts@gmail.com

\section{Roger Cammaerts}

Independent researcher, retired from the Natural and Agricultural Environmental Studies Department (DEMNA) of the Walloon Region, Belgium. Present address: 27, Square du Castel Fleuri, 1170 Bruxelles, Belgium. Tel.: 3226734969.

E-mail: rogercammaerts@gmail.com

Received: Sep. 17, $2020 \quad$ Accepted: Oct. 21, 2020

doi:10.5296/jbls.v11i2.17892 URL: https://doi.org/10.5296/jbls.v11i2.17892

\begin{abstract}
Numerosity ability is an important biological trait. We examined this trait in the ant Myrmica sabuleti over sixteen experimental works. A summary of the first nine works has been already published, and we provide here a summary of the last seven works. During these last-mentioned studies, we successively showed that ants natively possess a number line, acquire the notion of zero through experiences, have their counting ability only slightly affected, depending on the characteristics of the elements to count, can acquire single symbolism, can learn several symbols at the same time, can add up using learned symbols and can learn a symbol for zero and use it to add. Four of these abilities correspond to potential problems of numerical competence in humans. Humans have natively a number line, but rarely, they may suffer from not having it, what then reduces their skills in mathematics. Humans have to acquire the notion of zero in the course of their life, but this notion is not always correctly taught to children. Also, after having been learned to add and subtract using a concrete representation of numbers, human children should learn calculating using different representations, and finally without using any concrete representation. Humans can easily acquire symbolisms, and this ability should be employed without delay over the children's
\end{abstract}


education. No doubt that more knowledge on animal numerosity ability will be acquired in the future, maybe to be related to human numerical competence.

Keywords: counting, notion of zero, mental number line, numerical symbolism, operant conditioning

\section{Introduction}

\subsection{Aim of the Present Paper}

Animals' numerical ability, examined in invertebrates and vertebrates (references given below), is a very interesting subject that has its counterpart in humans. We already investigated on this subject working on ants as models, and recently published a summary of our first nine studies on this topic (Cammaerts \& Cammaerts, 2020b). These nine studies concerned the ants' notion and location of zero, adding and subtracting capability using visual as well olfactory cues (the cues being presented simultaneously or consecutively), the ants' possession of a left to right oriented number line, and their arrangement of amounts on that number line (Cammaerts \& Cammaerts, 2019a, b, c, d, e, f, g, 2020a; R. Cammaerts \& M.-C. Cammaerts, 2020). We then made seven more studies on the subject (Cammaerts \& Cammaerts, 2020c, d, e, f, g, h, i). The present paper intends to present a brief report of these last seven studies. They successively deal with the ants' native possession of a number line, acquisition of the notion of zero through experiences, counting accuracy when the characteristics of the amounts to count are changed, single numerical symbolism acquisition, multiple numerical symbolisms acquisition, use of numerical symbols for adding, as well as with the acquisition of a symbol for zero and its use for adding. Here below, in the introduction section, we summarize what is up to now known about the different numerical abilities we examined. Then, in a short method section, we recall our general experimental methods. Thereafter, we briefly report the experimental method specific to each study as well as the results of these studies. Finally, we comment our results and discuss about four possible related problems in humans.

\subsection{Short Bibliography About the Different Numerical Abilities Here Examined}

\subsubsection{Possessing a Number Line, Acquiring the Notion of Zero}

Investigation on the subject allows presuming that vertebrates have natively a left to right oriented mental number representation on a scale. For instance, a few days old chicks locate quantities on such a number line (Rugani et al., 2014; Rugani et al., 2015). Pigeons have also a mental number line (Brannon et al., 2001; Gallistel et al., 2001). Without having been trained, rhesus monkeys also appear to position quantities on a scale (Hauser et al., 2000) and Nieder \& Miller (2003) showed, by examining the neural response of rhesus monkeys, that they coded numerosity on a logarithmic scale. In humans, possessing a number line appears to be native and independent of the kind of reading or writing specific to civilization and education (see the instructive review of Rugani \& de Hevia, 2017).

Humans lately acquired the notion of zero over their history, some civilizations having never used a symbol for zero (Romans, Greeks). Humans also lately acquire this notion over their 
life. Three-year-old children do not perceive the zero as being a 'quantity' containing no element (Bialystok \& Codd, 2000). Four years old children acquire an understanding of zero as correct as that of the numbers (Bialystok \& Codd, 2000). It was observed that the cardinality understanding of zero and of small numbers develop independently from one another in infants 4 to 5 years old (Pixner et al., 2018). At the age of five, children's thinking begin to change (Bialystok \& Codd, 2000) and at about seven years old, most children consider that zero is a null amount inside a series of quantities (Wellman \& Miller, 1986). This corresponds to the statement of Nieder (2016) who pictures the humans' acquisition of the notion of zero through four main stages. Some non human vertebrates have been proved to have the notion of zero: the chimpanzee, Pan troglodytes (Biro \& Matsuzawa, 2001), rhesus monkeys, Macaca mulatta (Merritt et al., 2009), and the grey parrot, Psittacus erithacus, (Pepperberg \& Gordon, 2005). Concerning the invertebrates, the workers of the honeybee Apis mellifera were shown to detain a non abstract notion of zero by observing their reaction in front of a cue without element presented together with cues containing several elements (Howard et al., 2018). At our mind, up to now there existed no published research investigating on the acquisition of the notion of zero by young animals. Our work (Cammaerts \& Cammaerts, 2020d) on this interesting subject fills this gap.

\subsubsection{Counting independently of Numerosity Appearance}

This 'true counting' ability has been examined in several species by introducing changes in the representation of the cues to count. This has been done, for example, by changing cue sizes when assessing numerical ability in a spider (Rodríguez et al., 2015), by changing array configuration in pigeons (Emmerton \& Renner, 2006), chimpanzees (Tomonaga, 2008) and in gorillas and orangutans (Gazes et al., 2017). Keeping counting ability while changing the nature of food items and/or their size was demonstrated in dogs and wolves (Range et al., 2014), in a parrot (Pepperberg, 2012) and in an elephant (Irie et al., 2019). Moreover, brightness could be changed in mosquito fish (Agrillo et al., 2009). In rhesus and snow monkeys, it were color, shape, density, total surface, spatial arrangement, volume and total length that could be changed without affecting counting ability (Brannon \& Terrace, 1998, 2000; Cantlon \& Brannon, 2006; Jordan \& Brannon, 2006; Okuyama et al., 2015; Nieder et al., 2002; Flombaum et al., 2005). Workers of Apis mellifera have successfully demonstrated counting skills in front of cues with different shapes, dimensions, colors, displays or area appearances (Howard et al., 2019a, b). In 6- to 8-months-old human infants lacking experience with verbal counting, extraneous variables such as dot size and pattern could be used (Xu \& Spelke, 2000; de Hevia \& Spelke, 2010; Rugani \& de Hevia, 2017). At the age of four, size, color, location and array display could be used as extraneous variables for numbers 0 to 8 (Merritt \& Brannon, 2013). Of course, human adults were successfully experimented using amounts of elements of different sizes, areas, perimeters, densities, and displayed patterns (Cantlon \& Brannon, 2006; Merten \& Nieder, 2008). We intended to somewhat fill the gap between true counting and the masking effect of extraneous variables in ants (Cammaerts \& Cammaerts, 2020e). 


\subsubsection{Acquiring Numerical Symbolisms}

The honeybee could associate symbols such as shapes with 2 or 3 dots (Howard et al., 2019c). Pigeons could associate visual symbols with up to 4 elements (Xia et al., 2000) and a parrot (Psittacus erithacus) could associate vocal and visual symbols with up to 6 elements (Pepperberg, 2006a, b). Chimpanzees (Pan troglodytes) could associate Arabic numerals with 1 up to 7 or 9 elements (Murofushi, 1997; Matsuzawa, 1985; Beran, 2004) including the symbol 0 for "nothing" (Biro \& Matsuzawa, 2001). Rhesus monkeys (Macaca mulatta: Washburn \& Rumbauch, 1991), squirrel monkeys (Saimiri sciureus: Olthof et al., 1997), and capuchin monkeys (Cebus apella: Beran et al., 2008) could also associate Arabic numerals 0 to 9 with their corresponding number of food items.

\subsubsection{Using Learned Numerical Symbols for Making Arithmetic Operations}

Adding up and subtracting numbers of elements is an ability observed as early as in newborn chicks (Rugani et al., 2009). Dogs were shown to add and subtract numbers (West \& Young, 2002), and this ability was shown to be clearly detained by adult birds (Brannon et al., 2001; Garland \& Low, 2014), by rhesus (Hauser et al., 1996; Flombaum et al., 2005) as well as by snow monkeys (Macaca fuscata: Okuyama et al., 2015) and by chimpanzees (Rumbaugh et al., 1987). Honeybees have also been proved to be able to add up and subtract 1 element from 1 to 5 other ones (Howard et al., 2019a, b). The workers of the ant M. sabuleti could also make additions and subtractions, but only when perceiving the result of the operation during training (Cammaerts \& Cammaerts, 2019a, b, c). Several animal species can associate symbols with numbers of elements (see the above subsection). The further step should be the capability of making arithmetic operations by using learned numerical symbols. This was obtained in pigeons (Olthof, \& Roberts, 2000), in a grey parrot (Pepperberg, 2006a, 2012), in squirrel monkeys (Olthof et al., 1997) and in chimpanzees (Boysen \& Berntson, 1989). Also, the honeybee could learn to associate a symbol such as a given color with an addition or a subtraction to be made (Howard et al., 2019a). Making additions or subtractions using moreover a symbol for zero should be the still further step. However, animals not often detain the notion of zero. Honeybees detain it (Howard et al., 2018). Knowing what is zero for a parrot ( Psittacus erithacus) has been studied in several steps (Pepperberg, 1988; Pepperberg \& Gordon, 2005; Pepperberg, 2006a, b) what finally showed that it could make additions using the numbers 'zero' to 6. Chimpanzees (Pan troglodytes) concretely perceive 'zero' as being the lower quantity (Biro \& Matsuzawa, 2001). They could associate Arabic numbers with symbols including a symbol 0 for "nothing" (Biro \& Matsuzawa, 2001) and could make additions using Arabic numbers 0 to 4 (Boysen \& Berntson, 1989). Investigating on such potential abilities (i.e. making arithmetic operations using symbols, including the zero) in ants was thus our ultimate aim (Cammaerts \& Cammaerts, 2020h, i).

\subsection{Presentation of the Present Paper}

We first briefly describe our methods which are detailed in our previous successive papers. We then summarize our findings in seven subsections, each one corresponding to an already published work. In each of these subsections, we successively explain our experimental protocol providing photos different from those already published, and then present our 
quantitative results together with tables different from those already published.

\section{Methods}

\subsection{Collection and Maintenance of Ants}

We experimented on a total of twelve colonies of Myrmica sabuleti Meinert 1861 collected in abandoned quarries of the Aise valley, at Olloy/Viroin and Marchin (Belgium). These colonies were maintained in the laboratory in glass tubes half filled with water and set in trays which served as foraging areas. The ants were continuously provided with mealworms (their meat food) and sugar water delivered in small tubes plugged with cotton. The ambient temperature was $c a 20^{\circ} \mathrm{C}$, the humidity $80 \%$, and the lighting 330 lux while experimenting on and caring of the ants.

\subsection{Cues, Design and Protocol Common to the Seven Studies}

The visual cues presented to the ants were black or colored forms drawn on white paper, then cut and tied each one on a stand made of strong white paper $(2.5 \mathrm{~cm} \times 2.5 \mathrm{~cm}$ or $2 \mathrm{~cm} \times 2 \mathrm{~cm})$ and maintained vertically thanks to an orthogonally folded base. The cues used to test the ants were newly build ones, not those used for training them. During training, the cues to 'learn' were set near a reward (e.g. some food) and the cue to avoid was set far from any reward. The ants were trained in their foraging area and tested in a separate tray. Their responses were assessed by counting 20 times over 10 minutes the numbers of them approaching each kind of cue. The proportion of correct responses was established, and the recorded data was statistically analyzed using the non-parametric Wilcoxon test (Siegel \& Castellan, 1988). Schemas of each experimental design and protocol as well as statistical details can be found in Cammaerts \& Cammaerts (2020c, d, e, f, g, h, i). Photos of ants' training and testing are shown in Figures 1 to 7 , and numerical results are given in Tables 1 to 7 . All these illustrations differ from those published in the here above references.

\section{Results and Protocol Specific to Each Study}

\subsection{Ants Natively Detain a Number Line}

\subsubsection{Protocol}

The experiment was performed on only young ants (a few days to a few weeks old) of a large natural colony. These ants were maintained in six experimental nests, labeled A to F, and fed with sugared milk. They were trained to a number of green circles set near the food (the cue to memorize) versus a larger or a smaller number of green circles set far from the food (the cue to neglect). Colony A was provided with 1 green circle near the food and 3 ones far from it, colony B with 2 green circles near the food and 4 ones far from it, colony $\mathrm{C}$ with 3 green circles near the food and 5 ones far from it. Colony D was provided with 3 green circles near the food and 1 one far from it, colony $\mathrm{E}$ with 4 green circles near the food and 2 ones far from it, colony $\mathrm{F}$ with 5 green circles near the food and 3 ones far from it. Thus, colonies A, B, C received a small number to memorize and a larger one to neglect, while colonies $\mathrm{D}, \mathrm{E}, \mathrm{F}$ received a large number to memorize and a smaller one to neglect. The ants were tested (see Figure 1) in front of twice the number of green circles they should memorize, one on the left 


\section{Al Macrothink Institute $^{\mathrm{TM}}$}

and the other on the right of the smaller or larger number they should neglect. More precisely, the ants of colonies A, B, C were tested in front of 3, 4, 5 circles set between twice 1,2, 3 circles respectively, while ants of colonies D, E, F were tested in front of 1, 2, 3 circles set between twice 3, 4, 5 circles respectively. If the young ants possess a number line, they should react mostly to the 1,2, 3 circles located on the left of the $3,4,5$ ones, as well as to the $3,4,5$ circles located on the right of the 1,2, 3 ones.
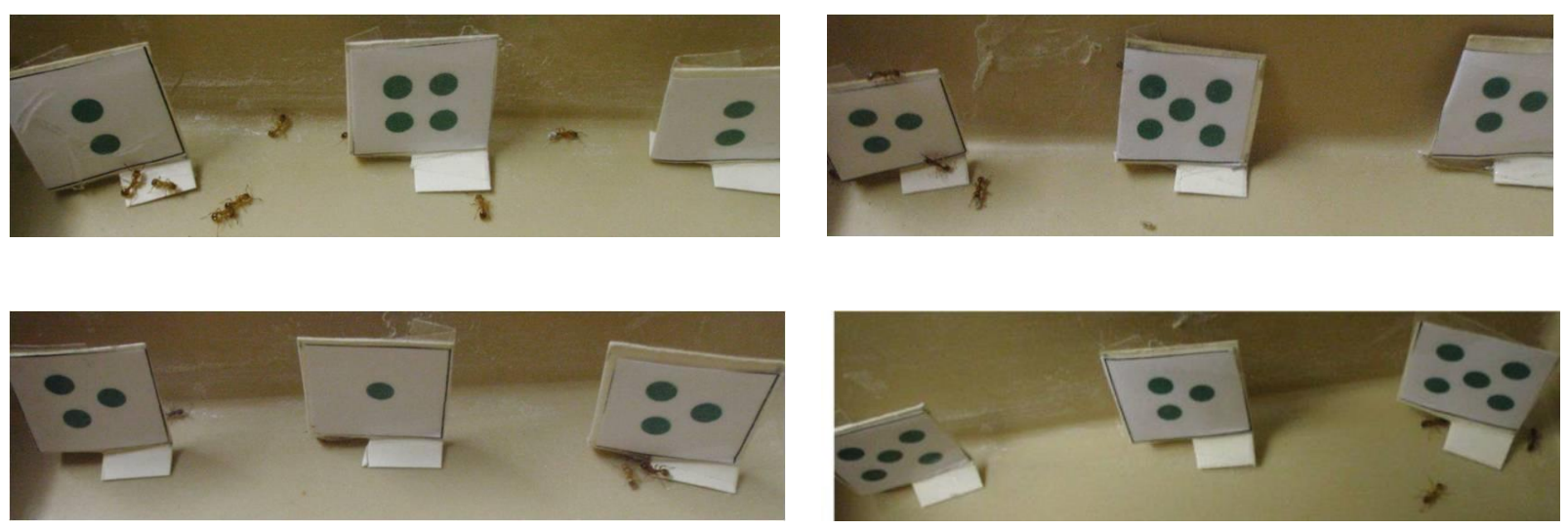

Figure 1. Views of experiments made on young ants for examining if they detain an oriented number line. The ants were trained to a small number of elements $v s$ a larger one or to a large number of elements $v s$ a smaller one. They reacted to the small number located on the left of the larger one (upper photos) and to the large number located on the right of the smaller one (lower photos). They thus natively, mentally positioned the small numbers on the left and the larger ones on the right side of a number line. Numerical results are given in Table 1.

\subsubsection{Results}

The numerical results are given in Table 1. The ants of colonies A, B, C approached essentially the 1,2, 3 circles located on the left of the 3, 4, 5 circles respectively. They located the smaller numbers on the left of the larger ones. The ants of colonies D, E, F approached essentially the 3,4,5 circles located on the right of the 1,2, 3 circles respectively. They located the larger numbers on the right of the smaller ones. Thus, young ants already detain a number line, on which the amounts are arranged from the left to the right. Ants possess natively a number line, like other animals examined until now about this topic. 
Table 1. Results of experiments made to know if young ants already have an oriented left to right number line

\begin{tabular}{|c|c|c|c|c|}
\hline \multicolumn{2}{|c|}{ Colony, training } & \multicolumn{3}{|c|}{ Testing in front of } \\
\hline & & on the left & in the middle & on the right \\
\hline $\mathbf{A}$ & 1 vs 3 & $1: 2.7$ & $3: 0.7$ & $1: 0.7$ \\
\hline B & 2 vs 4 & $2: 2.8$ & $4: 0.5$ & $2: 0.7$ \\
\hline $\mathbf{C}$ & 3 vs 5 & $3: 3.0$ & $5: 0.5$ & $3: 0.7$ \\
\hline $\mathbf{D}$ & 3 vs 1 & $3: 0.6$ & $1: 0.5$ & $3: 3.5$ \\
\hline $\mathbf{E}$ & 4 vs 2 & 4: 0.6 & $2: 0.6$ & 4: 3.1 \\
\hline $\mathbf{F}$ & 5 vs 3 & $5: 0.7$ & 3: 0 . & $5: 3.1$ \\
\hline
\end{tabular}

Young ants were trained to a small number of circles set near their food $v s$ a larger number set far from food (colonies A, B, C) or to a large number of circles set near the food $v s$ a smaller number set far from food (colonies C, D, E). The table gives the mean of 20 counts made during two tests $(\mathrm{N}=40)$. The tested young ants went mostly to the small number located on the left of the larger one, and to the large number located on the right of the smaller one. Thus, they natively mentally positioned the small amounts on the left and the larger ones on the right of an oriented number scale. Photos are shown in Figure 1.

\subsection{Ants Acquire the Notion of Zero Through Experiences}

\subsubsection{Protocol}

The experiments were conducted on only the young ants (of about a few weeks old) of a large natural colony, divided in four small colonies (A, B, C, D) maintained in artificial nests and provided with sugared milk delivered in plugged cotton tubes. The ants of colony A were trained to a black circle set near the food versus a white paper set far from it, those of colony B to a white paper set near the food versus a black circle set far from it, those of colony $\mathrm{C}$ to a black circle set near the food versus 'nothing' far from it, and those of colony D to nothing near the food versus a black circle set far from it. The ants were tested in front of the two cues presented during training. This allowed knowing if young ants already have the notion of zero. After that, each colony was provided with a tube filled of sugared milk and with an empty tube, set side by side at day 1 and apart from one another at day 2 . Thereafter, the ants of colonies $\mathrm{A}$ and B were trained firstly to a white paper set near the food versus a black circle set near an empty tube, and secondly to a white paper set near the food versus a black circle far from it. The ants of colonies C and D were trained firstly to 'nothing' near the food versus a black circle set near an empty tube, and secondly to 'nothing' near the food versus a black circle set far from it. These ants were then tested, in two testing sessions, in front of each pair (two in total) of cues they saw during training. This allowed examining if the young ants could acquire the notion of zero after having perceived the difference between a filled and an empty tube. 

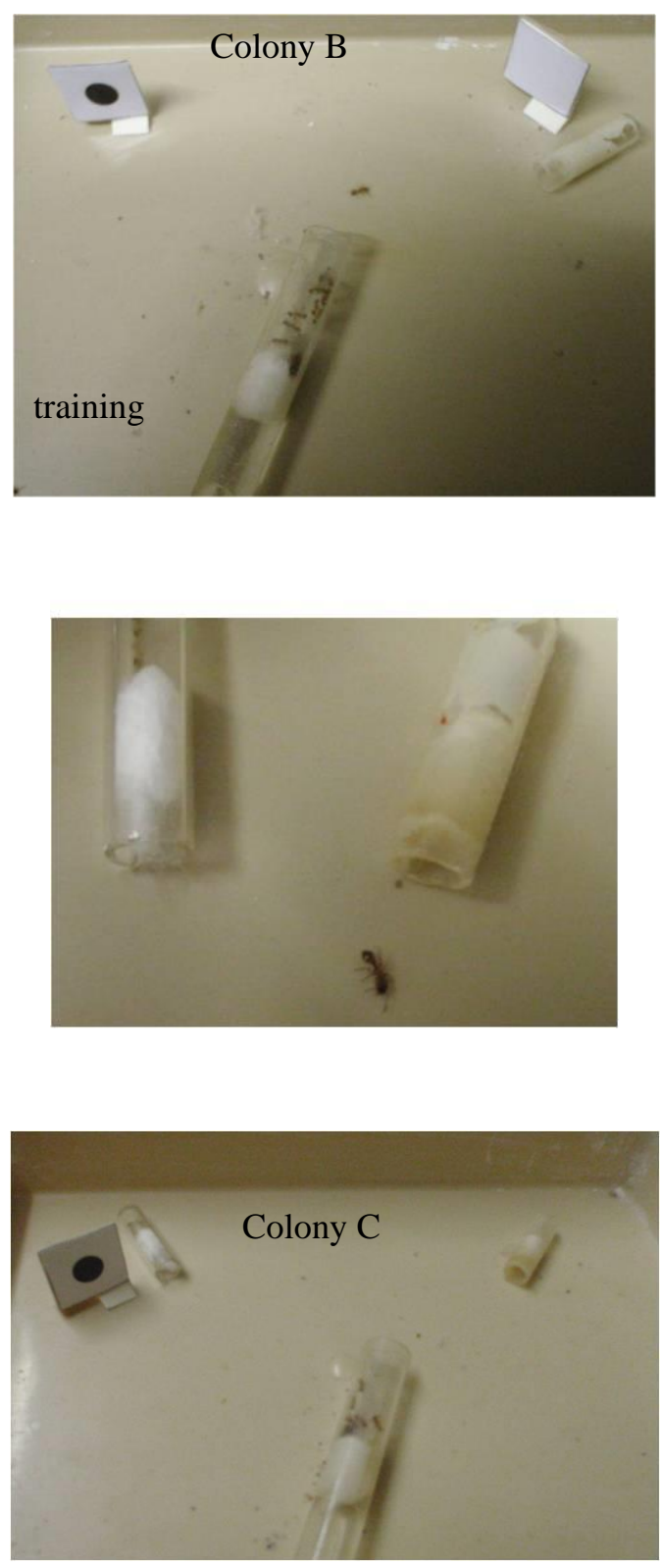

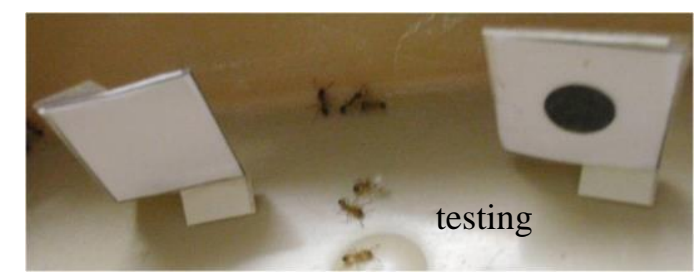

Trained to a white paper (zero) vs a black circle, the young ants did not react to the white paper.

They did not perceive zero as being a cue.

The young ants were provided with a filled tube

(on the right) and an empty one (on the left)

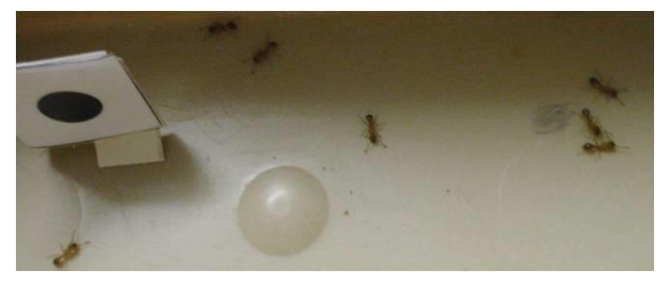

After that, trained to 'nothing' vs a black circle, the ants duly responded to 'nothing', perceiving it as a cue. They acquired the notion of zero

Figure 2. Views of experiments made on young ants for examining if they detain the notion of zero. They had not that notion (upper photos). Through experiences (middle photo), they acquired it (lower photos). Numerical results are given in Table 2.

\subsubsection{Results}

The numerical results are given in Table 2. During the first series of experiments, the ants could acquire conditioning to a concrete cue (a black circle) but not to a white paper or to 'nothing'. They thus have not the notion of 'nothing', of zero. During the second series of experiments, the ants could be conditioned to a white paper, as well as to 'nothing' (what has never been done), having thus, now, after their perception of a filled tube and an empty one, a notion of zero, and more exactly a concrete notion of zero. In wild, the ants acquire thus the notion of zero, of nothing, through experiences, in the course of their life. 
Table 2. Ants' acquisition of the notion of zero after having concretely experienced such a notion

\section{First series of experiments}

Colony, cues associated $v s$ not associated with food

Testing in front of the cues

associated not associated with food
A, a black circle $v s$ a white paper
3.5
1.0
B, a white paper $v s$ a black circle
1.2
1.0
C, a black circle $v s$ nothing
2.2
0.6
D, nothing $v s$ a black circle
1.6
2.1

\section{Second series of experiments}

Colonies

cues associated $v s$ not associated with food
Testing in front of the cues

associated not associated with food
A, B
a white paper $v s$ a black circle + an empty tube
2.9
0.7
a white paper $v s$ a black circle far from food
2.0
0.7
C, D
nothing $v s$ a black circle + an empty tube
nothing $v s$ a black circle far from food
0.8

The table gives the mean of 20 counts made during two tests on two colonies $(N=40)$. First series: the tested young ants appeared to be able to acquire conditioning to a black circle (a concrete cue) (colonies A and C) but not to a white paper or to 'nothing' (colonies B and D). They did not perceive the absence of a cue as an element. Second series: After having been accustomed to a filled and an empty food tube, the young ants were trained to a white paper or to 'nothing' vs a black circle set near an empty tube, then set far from food. When tested, they responded mostly to the white paper or to the 'nothing'; they have thus acquired a concrete notion of zero while simultaneously perceiving a filled and an empty tube. Photos of the experiments are shown in Figure 2. 


\section{Macrothink}

3.3 Influence of the Shape, Color, Size and Position of the Elements on the Ants'Counting

\subsubsection{Protocol}

The ants of four series of three colonies (labeled A, B, C; D, E, F; G, H, I; J, K, L) were trained to 1 vs 2 (A, D, G, J), 2 vs $3(\mathrm{~B}, \mathrm{E}, \mathrm{H}, \mathrm{K})$, and 3 vs 4 (C, F, I, L) blue squares, the number to memorize $(1,2,3)$ being set near the food, the other $(2,3,4)$ being set far from any reward (Figure 3, the upper photo for each kind of experiment). The ants were then tested in front of the two numbers of cues presented during training (i.e. that to memorize and that to avoid) but these cues had either their shape (square changed into circle, colonies A, B, C), color (blue changed into green, colonies D, E, F), size (small changed into large size, colonies $\mathrm{G}, \mathrm{H}, \mathrm{I}$ ), or position (their relative position on the stand, colonies $\mathrm{J}, \mathrm{K}, \mathrm{L}$ ) changed. Only one test is illustrated by a photo in Figure 3, for each kind of experiment. These training and testing sessions were performed to examine if the ants' counting is influenced by the appearance of the elements to count. In other words, the entire experiment aimed to know if ants truly 'count' elements or only retain a given figure.

\subsubsection{Results}

The numerical results are shown in Table 3. Whatever was the change of the characteristics of the cues to count between training and testing (change of shape, color, size, or position), the ants still statistically significantly discriminated 1 vs 2,2 vs 3, and 3 vs 4 of these elements. However, compared with their usual conditioning score of ca $80 \%$ (Cammaerts \& Cammaerts, 2014; Cammaerts \& Cammaerts, 2019a), their discrimination accuracy was a little affected when the characteristics of the cues to count were modified between training and testing (Cammaerts \& Cammaerts, 2020e). Changing the shape or the color of the elements to count only slightly affected the ants' counting ability (colonies A, B, C, D, E, F). When the size of the elements was changed (colonies G, H, I), the ants' counting accuracy was somewhat more impacted. A stronger, though not yet significant, effect was that caused by a change in the position of the elements to count between training and testing (colonies J, K, L). Such influence of the characteristics of the elements to count on the ants' counting ability is in agreement with the species' (M. sabuleti) visual perception, biology and navigation system (Cammaerts \& Cammaerts, 2014) 

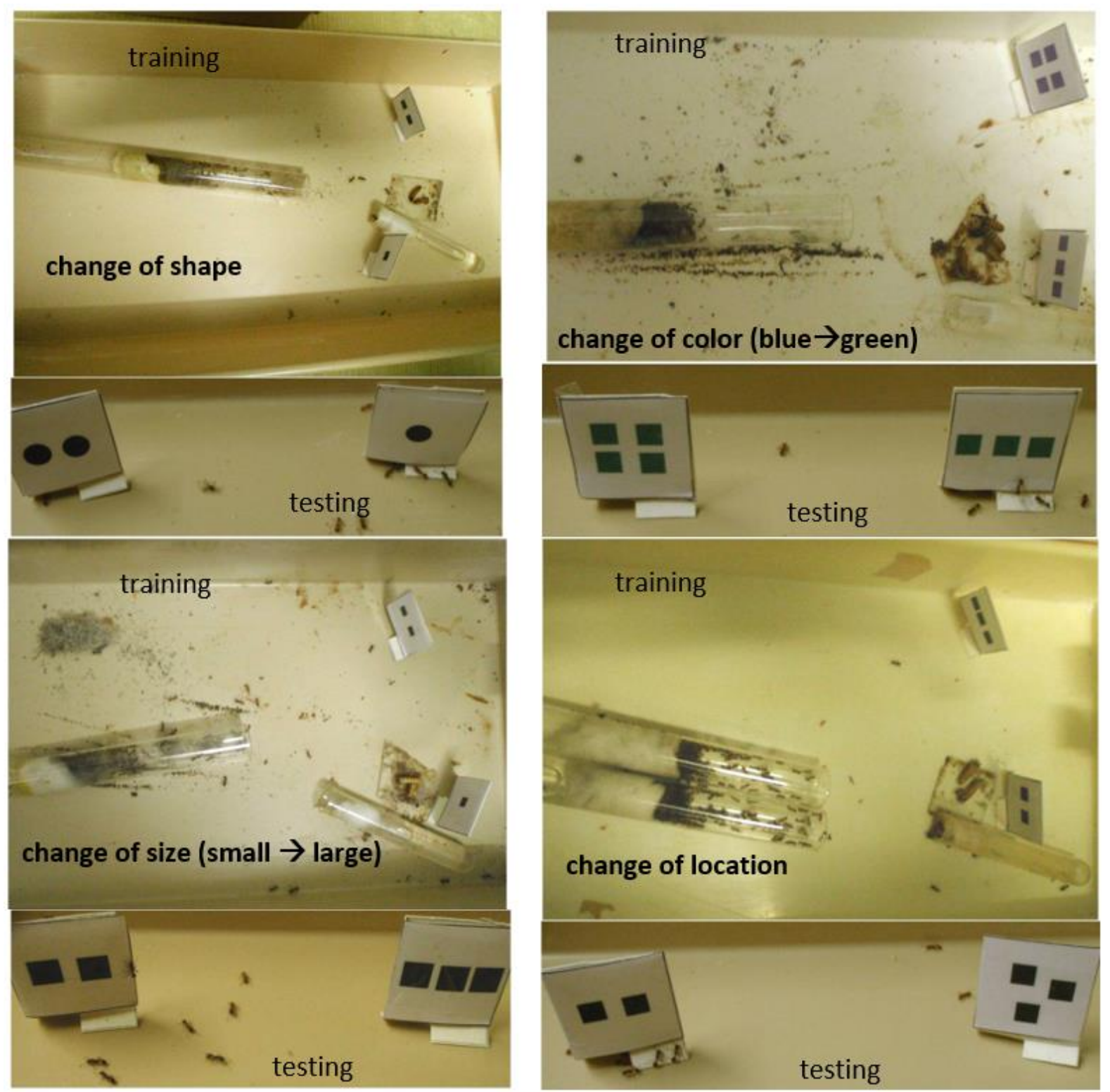

Figure 3. Views of experiments made to examine if the ants' counting ability is influenced by the characteristics of the elements to count. The ants could acquire conditioning (could count the elements) even if the shape, color, size or position of the elements were changed between training and testing. However, their counting accuracy was slightly, somewhat and rather well affected when the shape or color, the size, and the position respectively of the elements were changed between training and testing: see the numerical results presented in Table 3. 
Table 3. Influence of the shape, the color, the size and the position of elements on the ants' counting ability

\begin{tabular}{|c|c|c|c|c|}
\hline Colony & $\begin{array}{c}\text { Training } \\
\text { near }\end{array}$ & $\begin{array}{l}\mathrm{n}^{\circ} \text { of elements set } \\
\text { far from food }\end{array}$ & $\begin{array}{c}\text { Characteristic changed } \\
\text { between training and testing }\end{array}$ & $\begin{array}{l}\text { Testing: } \% \text { of correct } \\
\text { responses }\end{array}$ \\
\hline A & 1 & 2 & shape & $77.3 \%$ \\
\hline B & 2 & 3 & shape & $73.8 \%$ \\
\hline $\mathrm{C}$ & 3 & 4 & shape & $70.9 \%$ \\
\hline D & 1 & 2 & color & $77.2 \%$ \\
\hline $\mathrm{E}$ & 2 & 3 & color & $73.9 \%$ \\
\hline $\mathrm{F}$ & 3 & 4 & color & $70.8 \%$ \\
\hline G & 1 & 2 & size & $75.0 \%$ \\
\hline $\mathrm{H}$ & 2 & 3 & size & $72.2 \%$ \\
\hline I & 3 & 4 & size & $68.0 \%$ \\
\hline $\mathrm{J}$ & 1 & 2 & position & $72.3 \%$ \\
\hline $\mathrm{K}$ & 2 & 3 & position & $70.6 \%$ \\
\hline $\mathrm{L}$ & 3 & 4 & position & $66.2 \%$ \\
\hline
\end{tabular}

The correct cue was that located near the food during training. The ants could acquire conditioning and could thus discriminate and count the two presented cues. However, when the characteristics of the cues were not changed between training and testing, the ants' scores were somewhat higher, $c a 80 \%$ (e.g. Table 1). Thus, the ants could count elements whatever their shape, color, size and position, but the accuracy of 'true' counting was slightly influenced by the shape and the color of the elements, somewhat by their size, and rather well by their displayed position. These ants' sensitivities to the characteristics of visual elements are in agreement with the visual perception, the biology and the navigation system of the studied species. Photos of the experiments are shown in Figure 3.

\subsection{Ants Can Acquire Single Symbolism}

\subsubsection{Protocol}

The experiments were made on eight colonies: $\mathrm{A}$ and $\mathrm{B}, \mathrm{C}$ and $\mathrm{D}, \mathrm{E}$ and $\mathrm{F}, \mathrm{G}$ and $\mathrm{H}$ for the potential learning of a symbol for numerosities 1,2, 3 and 4 respectively. The ants' training can be seen in Figure 4, the two upper photos for each four symbols. Their testing is illustrated by only one photo for each symbol (Figure 4, the lower photo for each symbol). The ants of two colonies were trained to a number of black circles $(1,2,3$ or 4$)$ and its corresponding symbol, these two cues being set aside the meat food and the sugar water tube versus the number of black circles minus 1 (so: $0,1,2$ or 3 circles) set far from any reward. They were tested, in the course of three successive experiments, first in front of the number of circles set near the reward during training versus that set far from the reward, secondly in front of the corresponding symbol versus the number set far from any reward during training, and finally in front of the 'correct' number versus its corresponding symbol. These three experiments allowed examining if ants can associate a number with a given symbol. 

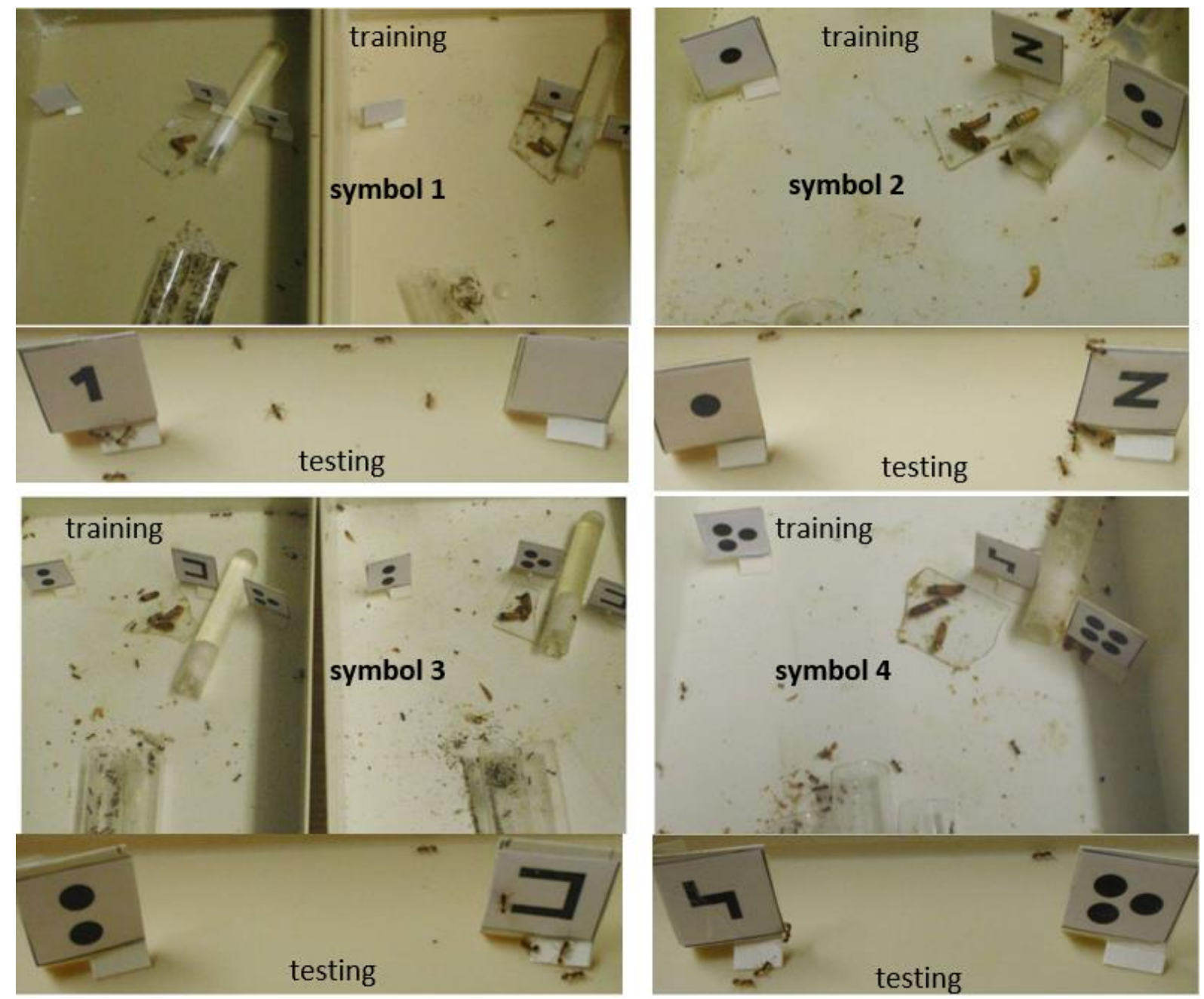

Figure 4. Views of experiments made to know if ants can acquire single symbolism. The ants were trained to a number of elements $(1,2,3$ or 4$)$ and a corresponding symbol set on one and the other side of the food (= the reward) $v s$ that number of elements minus 1 set far from food. When tested, the ants identically responded to the number of elements and to its corresponding symbol. They thus acquired single symbolism. Numerical results are given in Table 4

\subsubsection{Results}

The numerical results are given in Table 4. When tested, the ants of each two colonies trained to a number of circles and its corresponding symbol reacted more to the correct number than to that number minus 1 , more to the symbol than to the number minus 1 , and similarly to the number and its corresponding symbol. It was then successively checked if such behavior still occurred when the shape, color or size of the circles was changed, but these checking experiments are not here reported since the present paper is only a summary of our research. The ants' similarity of reaction to a number and to its symbol proved that they could acquire single symbolism thanks to operant conditioning. 
Table 4. Ants' acquisition of single symbolism

\begin{tabular}{|c|c|c|c|c|c|}
\hline \multicolumn{3}{|c|}{ Colonies $\mathrm{A}$ and $\mathrm{B}$} & \multicolumn{3}{|c|}{ Colonies $\mathrm{C}$ and $\mathrm{D}$} \\
\hline 1 circle: $72.4 \%$ & $v s$ & 0 circle: $27.6 \%$ & 2 circles: $72.9 \%$ & $v s$ & 1 circle: $27.1 \%$ \\
\hline symbol 1: $74.6 \%$ & $v s$ & 0 circle: $25.4 \%$ & symbol 2: $65.7 \%$ & $v s$ & 1 circle: $34.3 \%$ \\
\hline 1 circle: $48.1 \%$ & $v s$ & symbol 1: $51.9 \%$ & 2 circles: $48.7 \%$ & $v s$ & symbol 2: $51.3 \%$ \\
\hline \multicolumn{3}{|c|}{ Colonies $\mathrm{E}$ and $\mathrm{F}$} & \multicolumn{3}{|c|}{ Colonies $\mathrm{G}$ and $\mathrm{H}$} \\
\hline 3 circles: $69.3 \%$ & vs & 2 circles: $30.7 \%$ & 4 circles: $67.5 \%$ & vs & 3 circles: $32.5 \%$ \\
\hline symbol 3: $70.1 \%$ & $v s$ & 2 circles : $29.9 \%$ & symbol 4: $74.7 \%$ & $v s$ & 3 circles: $25.3 \%$ \\
\hline 3 circles: $48.7 \%$ & $v s$ & symbol 3: $51.3 \%$ & 4 circles: $49.4 \%$ & vs & symbol 4: $50.6 \%$ \\
\hline
\end{tabular}

The table gives the proportion of responses based on 20 counts made on two colonies $(\mathrm{N}=$ 40). Each time, the ants similarly reacted significantly more to the rewarded (correct) number of circles $(1,2,3$, or 4$)$ and its symbol than to that number minus one. They could thus acquire single symbolism. Photos of the experiments are shown in Figure 4.

\subsection{Ants Can Acquire Multiple Symbolisms}

\subsubsection{Protocol}

The ants of two colonies were at the same time trained to 1, 2, 3 and 4 black circles and to their corresponding symbol set aside, these four pairs of cues being set near a reward (meat food, sugar water tube, pieces of thyme and nest entrance) versus 5 circles set far from any reward (Figure 5, the two upper photos). The ants of the two colonies were then tested in the course of successive experiments, in front of each number of circles presented during training and 5 circles, in front of each corresponding symbol and 5 circles, and in front of each number of circles and their corresponding symbol (12 testing experiments: three kinds of experiments $x$ four different numbers) (Figure 5, the four lower photos).

\subsubsection{Results}

Numerical results are given in Table 5. When tested, the ants of the two colonies responded more to $1,2,3$ or 4 correct circles than to 5 circles, more to the symbol for $1,2,3$ and 4 circles than to 5 circles, and similarly to each number of circles and its corresponding symbol. They thus reacted identically to the different correct numbers and to their corresponding symbol sighted during training. They associated each correct number with their corresponding symbol, doing so simultaneously. They thus acquired multiple symbolisms. 


\section{Macrothink}
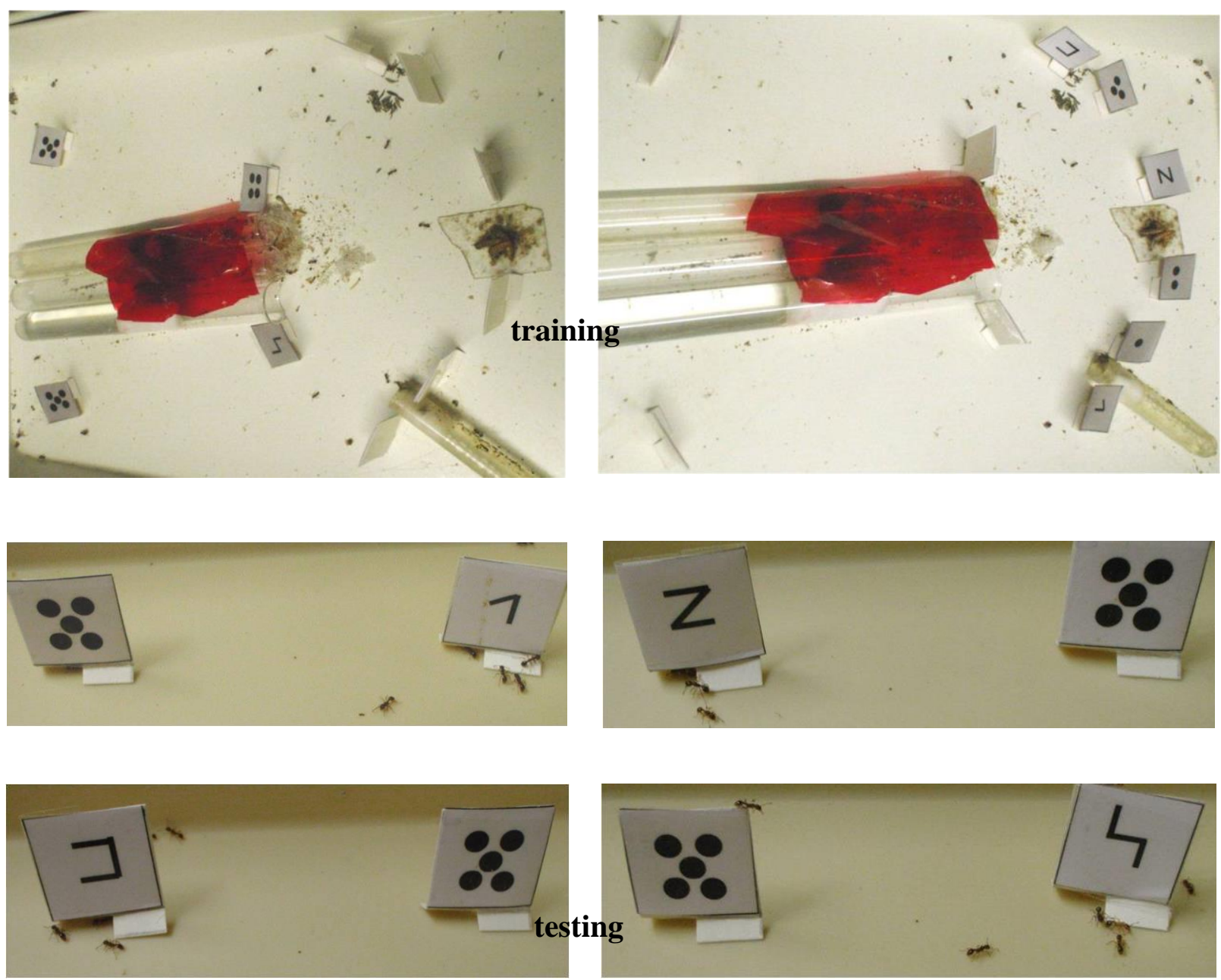

Figure 5. Views of experiments made to know if ants could acquire multiple symbolisms. The ants of two colonies were simultaneously trained to one dot and its symbol, to two dots and its symbol, to three dots and its symbol and to four dots and its symbol, each of these four paired cues being set near a reward $v s$ a cue with five dots set far from any reward (the two upper photos of the same colony are complementary, the colony being viewed from above under two different angles). When tested (the four lower photos), the ants duly reacted identically to the rewarded (correct) numbers and their respective symbol. They thus acquired multiple (i.e. four) symbolisms. Numerical results are given in Table 5. 
Table 5. Ants' acquisition of multiple symbolisms

\begin{tabular}{|c|c|c|c|c|c|}
\hline 1 circle: $78.5 \%$ & $v s$ & 5 circles: $21.5 \%$ & 2 circles: $71.9 \%$ & $v s$ & 5 circles: $28.1 \%$ \\
\hline symbol 1: $73.2 \%$ & $v s$ & 5 circles: $26.8 \%$ & symbol 2: $76.1 \%$ & $v s$ & 5 circles: $23.9 \%$ \\
\hline 1 circle: $52.6 \%$ & $v s$ & symbol 1: $47.4 \%$ & 2circles: $53.9 \%$ & $v s$ & symbol 2: $46.1 \%$ \\
\hline 3 circles: $69.8 \%$ & $v s$ & 5 circles: $30.1 \%$ & 4 circles: $68.0 \%$ & $v s$ & 5 circles: $32.0 \%$ \\
\hline symbol 3: 78.8\% & $v s$ & 5 circles: $21.2 \%$ & symbol 4: $74.0 \%$ & $v s$ & 5 circles: $26.0 \%$ \\
\hline 3 circles: $47.1 \%$ & $v s$ & symbol 3: $52.9 \%$ & 4 circles: $47.5 \%$ & $v s$ & symbol 4: $52.5 \%$ \\
\hline
\end{tabular}

The table gives the proportion of correct responses based on 20 counts made on two colonies $(\mathrm{N}=40)$. The ants of the two colonies could simultaneously associate the numbers $1,2,3$, and 4 to their corresponding symbol since they similarly reacted to these numbers and symbols. They thus acquired multiple symbolisms thanks to operant conditioning. Photos are shown in Figure 5.

\subsection{Ants Can Use Learned Numerical Symbols for Making Additions}

\subsubsection{Protocol}

Firstly, the ants of colonies $\mathrm{A}$ and $\mathrm{C}$ were trained to two numbers of black circles and the ants of colonies B and D were trained to three numbers of such circles, as well as each time to their corresponding symbols, these cues being set aside a reward (the meat food, the sugar water tube, pieces of thyme) versus a white paper set far from any reward (Figure 6, upper left photo). They were then tested, in the course of series of experiments, first in front of each two or three numbers of circles presented during training versus a white paper, then in front of each two or three corresponding symbols versus a white paper, and finally in front of each two or three numbers versus their corresponding symbol (Figure 6, lower left photos). These thirty experiments [two series of three experiments on colonies A and C $(2 \times 3 \times 2)+$ three series of three experiments on colonies $B$ and D ( $3 \times 3 \times 2)$ ] allowed checking if the ants could acquire the proposed symbolisms.

After that, a second training was conducted: the ants of the four colonies were moreover provided with two numbers of circles set side by side on one side of the nest entrance and with their two corresponding symbols set side by side on the other side of the nest entrance (Figure 6, upper right photo). They were then tested, in the course of two experiments, in front of the correct added numbers and of two wrong numbers of circles, then in front of a symbol corresponding to the correct added numbers and of two wrong symbols (Figure 6, lower right photos). These eight experiments (two on each four colonies) allowed examining if the ants could make the suggested additions by using the numbers of circles as well as the corresponding symbols. 

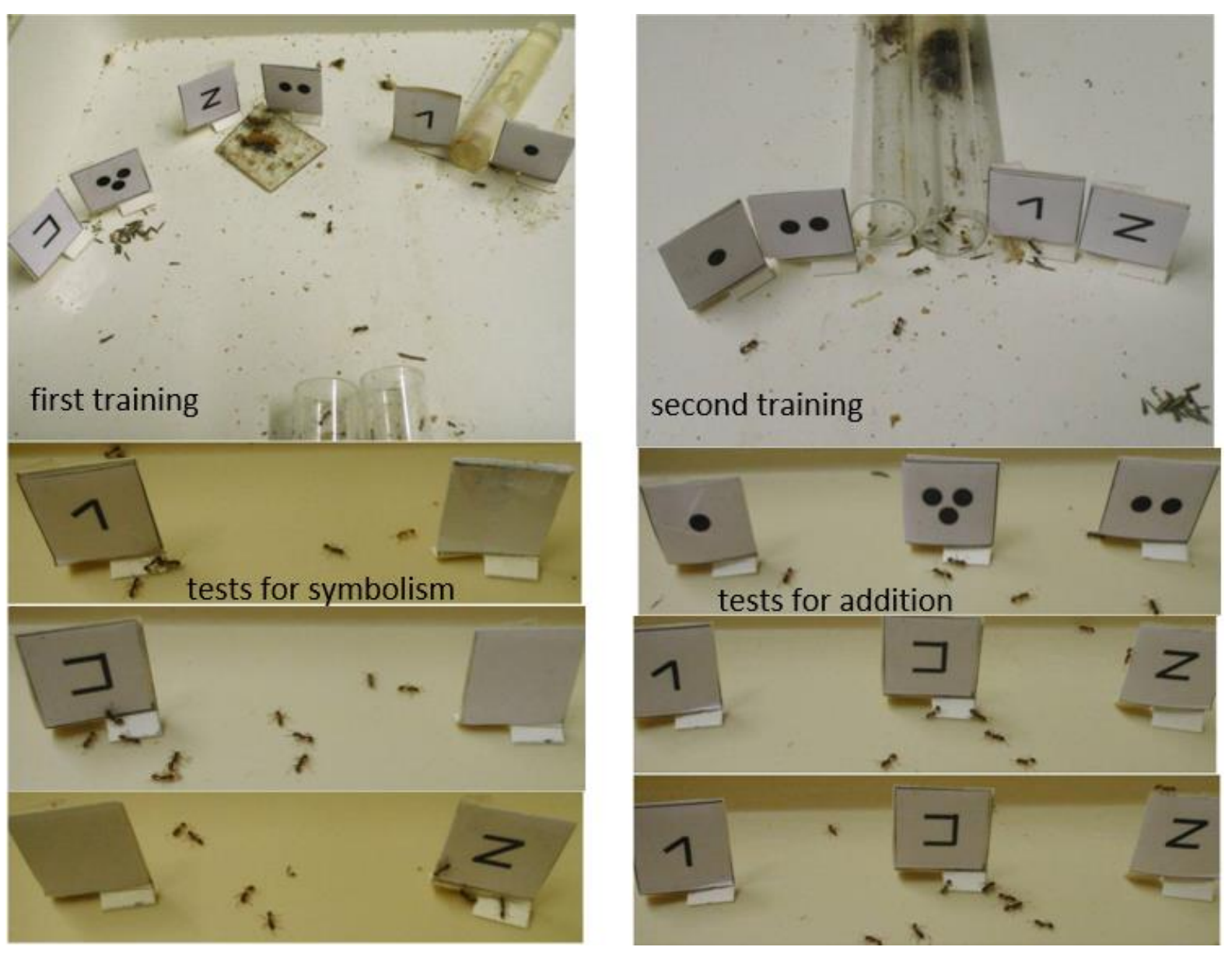

Figure 6. Views of experiments made on colony B in order to see if ants could use learned symbols for making additions. Only the experiments relative to one kind of addition are here illustrated, three other additions having also been examined. The ants were firstly trained to two numbers of elements and to their corresponding symbols (upper left photos), and the ants' symbolism acquisition was checked (lower left photo). The ants were then trained in front of a suggested addition using the numbers of elements as well as their symbols (upper right photo). The tested ants could make the addition using either the numbers or their symbols (lower right photos). Numerical results are given in Table 6.

\subsubsection{Results}

The numerical results are given in Table 6. During their first testing, the ants of each colony similarly responded to the numbers of circles and their corresponding symbols presented during training. They thus acquired the required multiple symbolisms. During their second testing, the ants of each colony reacted mostly to the correct added numbers of circles, as well as to the correct corresponding symbols. They thus duly added up the numbers as well as the symbols presented side by side, i.e. they made the suggested addition using the numbers of elements as well as their corresponding symbols. 
Table 6. Ants' use of learned symbols for making a suggested addition

\section{Colony A}

1 circle: $72.0 \% \quad$ vs $\quad 0$ circle: $28 \%$

symbol 1: $72.1 \%$ vs 0 circle $: 27.0 \%$

symbol 1: $50.0 \%$ vs 1 circle: $50.0 \%$

2 circles: $70.9 \% \quad$ vs $\quad 0$ circle: $27.3 \%$

symbol 2: $72.7 \%$ vs 0 circle: $27.3 \%$

symbol 2: $50.7 \%$ vs 2 circles: $49.3 \%$

1: $18.3 \%$ vs $2: 61.7 \%$ vs 0 circle: $20.0 \%$

symbol 1: $28.8 \%$ vs $2: 67.3 \%$ vs $0: 3.8 \%$

\section{Colony C}

2 circles: $80.9 \% \quad$ vs 0 circle: $19.1 \%$

symbol 2: $72.5 \% \quad$ vs 0 circle $: 27.3 \%$

symbol $2: 48.3 \% \quad$ vs 2 circles: $51.7 \%$

4 circles : $80.6 \% \quad$ vs 0 circle $: 19.4 \%$

symbol $4: 74.6 \%$ vs 0 circle $: 25.4 \%$

symbol $4: 50.0 \% \quad$ vs 4 circles: $50.0 \%$

2: $13.2 \%$ vs $4: 67.9 \%$ vs 2 circles: $18.9 \%$

symbol 2: $13.2 \%$ vs $4: 67.6 \%$ vs $2: 19.1 \%$

\section{Colony B}

1 circle: $76.5 \% \quad$ vs 0 circle: $23.5 \%$

symbol 1: $82.9 \% \quad$ vs 0 circle $: 17.1 \%$

symbol 1: $49.0 \%$ vs 1 circle: $51.0 \%$

2 circles: $78.1 \% \quad$ vs $\quad 0$ circle: $21.9 \%$

symbol 2: $78.0 \% \quad$ vs 0 circle: $22.0 \%$

symbol 2: $48.2 \%$ vs 2 circles: $51.8 \%$

3 circles: $71.9 \% \quad$ vs $\quad 0$ circle: $28.1 \%$

symbol 3: $72.9 \% \quad v s \quad 0$ circle: $27.1 \%$

symbol 3: $48.6 \%$ vs 3 circles: $51.4 \%$

1: $7.1 \%$ vs $3: 73.2 \%$ vs 2 circles: $19.6 \%$

symbol 1:13.1\% vs 3: $65.6 \%$ vs $2: 21.3 \%$

\section{Colony D}

3 circles: $74.5 \% \quad$ vs $\quad 0$ circle: $25.5 \%$

symbol 3: $80.4 \%$ vs 0 circle: $19.6 \%$

symbol 3: $48.2 \%$ vs 3 circles: $51.8 \%$

1 circle: $71.0 \% \quad$ vs $\quad 0$ circle: $29.0 \%$

symbol 1: $78.4 \% \quad$ vs $\quad 0$ circle: $21.6 \%$

symbol 1: $50.8 \% \quad$ vs $\quad 1$ circle: $49.2 \%$

4 circles: $81.5 \% \quad$ vs 0 circle: $18.5 \%$

symbol 4: $82.1 \% \quad v s \quad 0$ circle: $17.9 \%$

symbol 4: $48.5 \% \quad$ vs 4 circles: $51.5 \%$

3: $14.1 \%$ vs $4: 61.3 \%$ vs 1 circle: $24.6 \%$

symbol 3: $6.7 \%$ vs 4: $83.3 \%$ vs 1: $10.0 \%$

The table gives the proportion of correct responses based on 20 counts $(\mathrm{N}=20)$. Each time, the ants could acquire the presented multiple symbolisms and make the suggested addition (colonies A, B, C, D: respectively $1+1,1+2,2+2,3+1$ ) using the numbers as well as the symbols presented side by side. Photos are shown in Figure 6. 


\subsection{Ants Can Learn a Symbol for Zero and Use It for Making Additions}

\subsubsection{Protocol}

The ants of four colonies were firstly trained to a number of black circles $(1,2,3$ and 4 respectively) as well as to its corresponding symbol, the two cues being set aside the sugar water tube versus 5 black circles set far from any reward. At the same time, they were also trained to a white paper (= zero circle) and a symbol for zero, these two cues being set near the meat food versus the 5 circles set far from food (Figure 7, upper left photo). The ants of the four colonies were then tested in front of the number of circles sighted during training versus 5 circles, in front of its corresponding symbol versus 5 circles, in front of the number of circles versus its symbol, in front of a blank paper versus 5 circles, in front of the symbol for zero versus 5 circles, and in front of the symbol for zero versus a blank paper (Figure 7, lower left photos). These six tests allowed checking if ants duly acquired the required symbolisms.

After that, the ants of the four colonies were moreover provided with a number of black circles (again respectively 1, 2, 3 and 4) and a blank paper, the two cues being set on the left of the nest entrance, as well as at the same time with the symbol for the number of circles (i.e. respectively the symbol for 1, 2, 3 or 4) and the symbol for zero, these two cues being set on the right of the nest entrance (Figure 7, upper right photo). The ants of the four colonies were then tested in front of 5 circles, of the number of circles provided during training, and of a white paper, then, in front of 5 circles, of the symbol corresponding to the number of circles seen during training and of the symbol for zero (Figure 7, lower right photos). These two tests allowed knowing if the ants could make a suggested addition (i.e. $1+0,2+0,3+0,4+0$ ) using the numbers of circles and using the corresponding symbols.

\subsubsection{Results}

The results are numerically presented in Table 7. During their first testing session, the ants of each colony responded similarly to the number of circles and its corresponding symbol, as well as similarly to the blank paper and to its corresponding symbol, to which they had been trained. They duly acquired the two required symbolisms. During their second testing session, these ants approached significantly mostly the number of circles as well as the symbol which corresponded to the result of the suggested addition $(1+0=1,2+0=2,3+0=3,4+0=4)$. They thus duly added up a number of elements + zero element $=$ the number of elements, as well as a symbol for a number + a symbol for zero $=$ the symbol for the number. They could learn a symbol for zero, and could use it for adding, just like they could use numerical symbols (see the previous experiment related here above). 


\section{Macrothink}
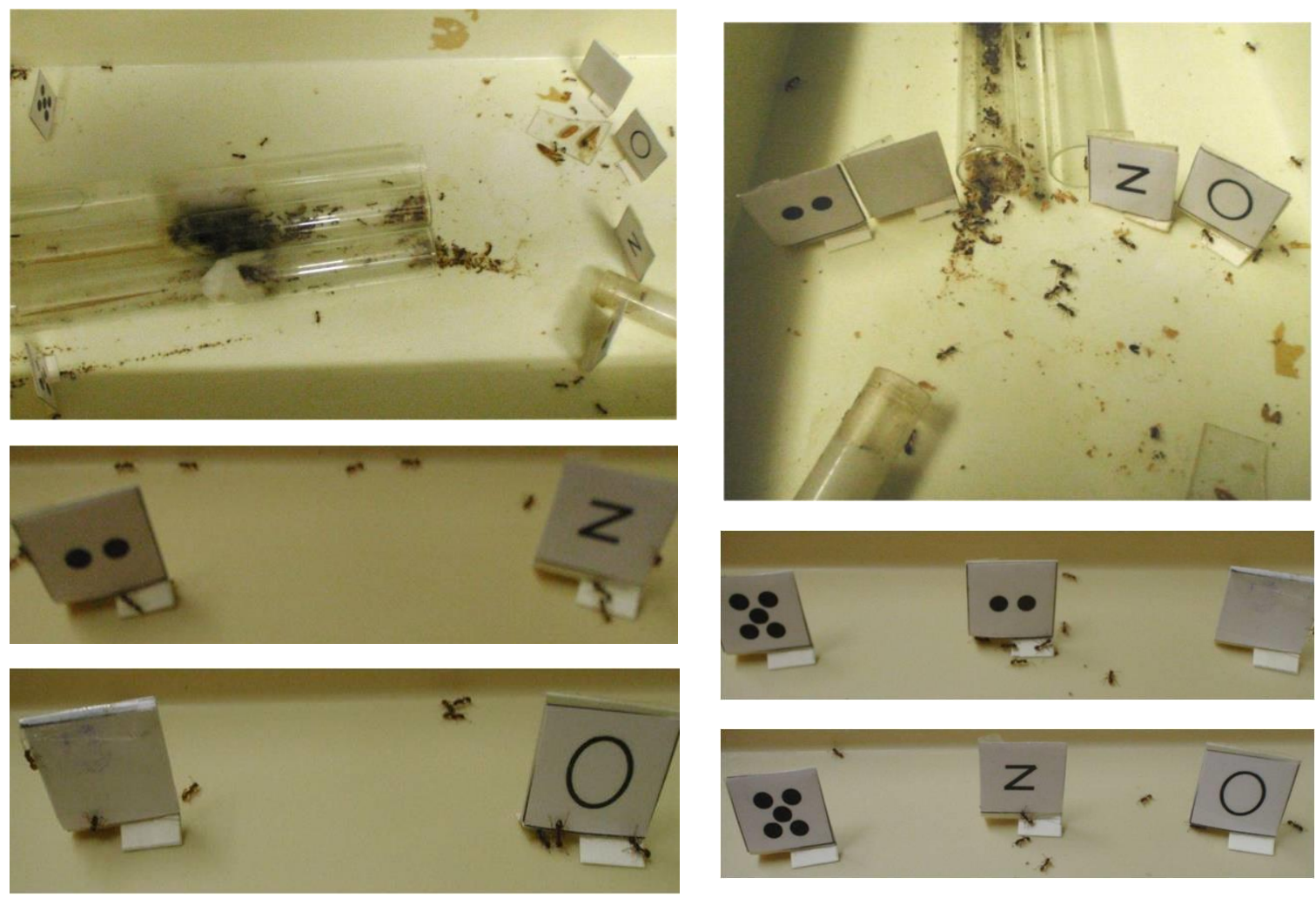

Figure 7. Views of experiments performed for knowing if ants could learn a symbol for zero and use it to add up. Only the experiments relative to one addition are here illustrated; three other series of experiments relative to three other additions were also performed. The ants were firstly trained to a number of elements and its corresponding symbol as well to zero element and its corresponding symbol (upper left photos), and the ants' acquisition of these symbolisms was checked (lower left photo). The ants were then trained in front of a suggested addition represented by the numbers of elements as well as by the corresponding symbols (upper right photo). When tested, the ants could make the addition using either the numbers or their symbols (lower right photos). Numerical results are given in Table 7. 
Table 7. Ants' learning of a symbol for zero and using of it for adding up

\section{Colony A}

1 circle: $78.9 \% \quad$ vs $\quad 5$ circles: $21.1 \%$

symbol 1: $76.5 \%$ vs 5 circles: $23.5 \%$

symbol 1: $52.8 \%$ vs 1 circle: $47.2 \%$

0 circle: $85.2 \%$ vs 5 circles: $14.8 \%$

symbol 0: $78.3 \% \quad$ vs 5 circles: $21.7 \%$

symbol 0: $47.2 \% \quad$ vs 0 circle: $52.8 \%$

5: $5.0 \%$ vs $1: 63.3 \%$ vs 0 circle: $31.7 \%$

5 circles: $10.6 \%$ vs sy $1: 72.7 \%$ vs sy0:16.7\%

\section{Colony B}

2 circles: $77.1 \%$ vs 5 circles: $22.9 \%$

symbol 2: $75.8 \%$ vs 5 circles: $24.2 \%$

symbol 2: $51.6 \%$ vs 2 circles: $48.4 \%$

0 circle: $74.1 \% \quad$ vs $\quad 5$ circles: $25.4 \%$

symbol 0: $85.9 \%$ vs 5 circles: $14.1 \%$

symbol 0: $50.0 \% \quad v s \quad 0$ circle: $50.0 \%$

5: $12.8 \%$ vs $2: 66.0 \%$ vs 0 circle: $21.3 \%$

5 circles: $8.2 \%$ vs sy2:77.6\% vs sy0:14.3\%

\section{Colony C}

3 circles: $82.6 \% \quad$ vs 5 circles: $17.4 \%$

symbol 3: $84.6 \%$ vs 5 circles: $15.4 \%$

symbol 3: $51.5 \% \quad$ vs 3 circles: $48.5 \%$

0 circle $: 86.7 \%$ vs 5 circles: $13.3 \%$

symbol 0: $85.4 \%$ vs 5 circles: $14.6 \%$

symbol 0: $50.0 \%$ vs 0 circle: $50.0 \%$

5: $16.1 \%$ vs $3: 67.9 \%$ vs 0 circle: $16.1 \%$

5 circles: $13.7 \%$ vs sy3: $70.6 \%$ vs sy0: $15.7 \%$

\section{Colony D}

4 circles: $78.0 \% \quad$ vs $\quad 5$ circles: $22.8 \%$

symbol 4: $84.5 \% \quad$ vs $\quad 5$ circles: $15.5 \%$

symbol 4: $51.1 \% \quad$ vs 4 circles: $48.9 \%$

0 circle: $80.6 \% \quad$ vs 5 circles: $19.4 \%$

symbol 0: $77.9 \% \quad$ vs $\quad 5$ circles: $22.1 \%$

symbol 0: $48.8 \% \quad$ vs 0 circle: $51.2 \%$

5: $13.2 \%$ vs $4: 60.4 \%$ vs 0 circle: $26.4 \%$

5 circles: $10.3 \%$ vs sy $4: 74.1 \%$ vs sy0: $15.5 \%$

The table gives the proportions of correct responses based on 20 counts $(\mathrm{N}=20)$. Each time, the ants could acquire the proposed symbolisms including that for zero, and could make the suggested addition (colonies A, B, C, D: respectively $1+0,2+0,3+0,4+0$ ) using the numbers as well as the symbols presented side by side. Photos are shown in Figure 7.

\section{Discussion}

In this section, we first comment our findings in the order they have been related, then we present four associated potential problems that may appear in human's numerical competence, and thirdly, we propose a personal conclusion.

\subsection{Comments}

The native characteristic of the number line. Ants natively possess a mental number line. Human infants at the age of 7-8 months, before experiencing language, symbolism and 
measurement, tend to relate numerical magnitude with spatial length, what is the essence of a mental number line (de Hevia \& Spelke, 2010). Even neonates relate numbers and durations to spatial length when these dimensions vary in the same direction, although not when in opposite directions (de Hevia, Izard et al., 2014). At the age of 7 months, infants prefer increasing numerical sequences than decreasing ones when they are displayed in a left to right spatial orientation, as well as decreasing sequences than increasing ones when under the reverse orientation (de Hevia, Girelli et al., 2014). They have a native number line, what is still observed in preschool 3 to 5 year old children who intuitively relate numbers and length (de Hevia et al., 2012). It appears that the precision of these preschool infants in representing amounts on a number line predicts their later mathematical performance (Mazzocco et al., 2011). The human number mapping is initially logarithmic, but mathematical education induces a shift towards a linear mapping (Dehaene et al., 2008). Such a shift from logarithmic to linear representation of amounts was examined in the course of infants' education (Laski \& Siegler, 2007; Siegler et al., 2009). The acquisition of a precise number representation is 'a mental revolution, unique to Homo sapiens', and is the first step to higher mathematics (Dehaene, 2011, p 260).

The notion of zero. Ant workers acquire the notion of zero thanks to individual experiences. Here, we presented to ants an empty and a filled sugar water tubes in order to learn them the notion of 'nothing', of zero. In the wild, the ants could acquire that notion when perceiving the absence of water or of food, the departure of male and/or female individuals, the death of one of their queens, etc. For animals living in groups, zero may be learned through the departure or the death of the pack leader. Each time, the brutal absence of a vital or important element may bring the notion of zero to an animal. The acquisition of the notion of zero may thus occur at different moments of an individuals' life, potential differences appearing therefore among individuals and possibly between populations. Brysbaert (1995) found that humans take more time to read the numeral 0 than expected on the basis of their numbers representation. Zero learning differs from that of true amounts, but finally, zero is perceived as being the smallest quantity located on the humans' mental number line (Pinhas \& Tzelgov, 2012). In fact, human children lately acquire a correct notion of zero ( $c a$ at the age of four: Bialystok \& Codd, 2000; Nieder, 2016) and do not represent it by a symbol before the age of about six (Wellman \& Miller, 1986).

True counting. We found that ants went on duly counting elements when their shape, color, size or location was changed, although their counting accuracy was then lower and this, when only one characteristic of the elements has been changed. True counting was demonstrated in honeybees. Gross et al. (2009) experimentally proved that they truly counted numbers of elements independently of their color, configuration, area, perimeter or the illusory contours they formed. However, bees could apply their learning of distinguishing 2 from 3 to that of 3 from 4, but not to that of 4 from 5 and 5 from 6. Bortot et al. (2019) showed that bees effectively could be learned to count numbers of black circles whatever the size and pattern configuration of these dots, the correct number being the smaller or the larger one. However, the latter ability was significant for 2 vs 3 and 3 vs 2 but not for $3 v s 4$ and 4 vs 3 . The bees' true counting ability may thus be limited to 4 items. Irie et al. (2019) showed that an elephant 
could distinguish two different amounts of elements without being affected by the numerical distance, the size or the ratio of these amounts or by their ratio. However, this finding was obtained in only one individual with a proportion of correct responses equaling $66.8 \%$, what was a rather low performance. Jordan and Brannon (2006) showed that rhesus monkeys truly counted items independently of their non-numerical characteristics. In the same way, Ditz and Nieder (2016) demonstrated that crows (Corvus corone) correctly counted elements even if the elements presented during testing differed by their size and display from those used for training. In contrast, Tomonaga (2006) showed that pattern characteristics of the dot display somewhat influenced the chimpanzees' counting ability though they still significantly perceived the difference between the number of dots. This result recalls what we found on ants.

Symbolism acquisition and use. We have checked (but reported the information only in the paper relative to symbolism acquisition (Cammaerts \& Cammaerts, 2020f) and not in the present summary) that ants could associate numbers with symbols, even if the color, shape or size of the elements representing the numbers were changed. The ants appeared to better make the proposed additions when using the symbols than when using the numbers. Animals' symbolism acquisition may thus be easy since useful, and the list of species able to acquire numerical symbolism given in the introduction section may be longer. Indeed, up to now, using numerical symbolism including the zero for making additions has been found only in chimpanzees (Pan troglodytes) (Boysen \& Berntson, 1989), and in rhesus monkeys (Macaca mulatta) (Washburn \& Rumbaugh, 1991). However, it is plausible that such ability is detained by other animals not yet investigated as for this topic since the use of symbols is not a complex task but an easy one, a task simplifying, for example, the counting and calculation of amounts. In fact, species living in groups use visual, acoustical, olfactory and tactile (i.e. (biological) signals to communicate. Each of these social signals can be considered as being a symbol with its own signification and usefulness. There is a gap between numerical symbolisms acquired by humans through education, and the symbolism learned through conditioning by animals. The numerical competence of humans passes from an iconic to an indexical and finally to a symbolic stage, but animals are constrained to an indexical level (Nieder, 2009). Let us add that among all the animal species, even if they make use of several symbolisms in their day-to-day life, no one has been shown to have spontaneously created a numerical symbolism. Only the human species did so (Howard et al., 2019b).

\subsection{Related Events in Humans}

Humans' innate possession of a number line is validated by the consequence of two health impairments. Firstly, brain damage (hemi-spatial neglect) affects the representation of numbers on a mental number line (Zorzi et al., 2002). Secondly, children with normal intelligence but difficulties when starting to learn simple mathematical operations appear to be deficient as for the possession of a number line (dyscalculia) Such children are treated thanks to a particular training program and make progress in mathematical learning in the course of their acquisition of a number line (Kucian et al., 2011).

Of course, infants must acquire true counting for going on with mathematical education. The 
notion of numbers and the elementary arithmetical operations should be learned to infants during their most adequate life period, using a concrete representation of the numbers, and only one (always the same) representation until the infants could correctly count, add up and subtract. Thereafter, some short time periods should be devoted to extent the acquired learning to several kinds of number representations in order to soon obtain the children's counting, adding up and subtracting abilities without the help of concretely representing the numbers. At that time, the notion of zero should be included in the infants' education.

Zero is a non-native notion that has to be explained to infants. Unfortunately, this learning is not always efficiently made (Wheeler \& Feghali, 1983). More attention should be paid to this important point of the humans' education, childhood being the best life period for learning the signification of the zero. Attention should be paid to the manner according to which this learning would be optimally performed.

Concerning numerical symbolism (and any other symbolisms), it appears that it can easily be acquired by several animal species, and in fact also by humans at any age. Even more, many tasks (mathematical and other ones) could be easier and more rapidly performed using symbolism. We advice to think as soon as possible to learn symbols to infants, to not neglect this small but very important part of the humans' education. The use of symbolism is valid for nearly all the cognitive topics, e.g. music, mathematics, writing, driving, visual communication, etc... In other words, learning symbolisms to infants must not be delayed; learning and using symbols is easily acquired by them, and makes plenty of their activities easier and more rapid.

\subsection{Conclusion}

Studying animals' numerosity abilities shows that humans are not the only species possessing such abilities. Vertebrates and invertebrates also can count, add, subtract amounts, have a native number line, may acquire the notion of zero, and can acquire symbolisms, all this being beneficial for their life. Moreover, such studies point out several numerical particularities in animals which exist in humans and are important for the mathematical learning of the latter. Animals serve as biological models for better understanding human biology, and this includes numerical abilities.

\section{References}

Agrillo, C., Dadda, M., Serena, G., \& Bisazza, A. (2009). Use of number by fish. PLoS ONE, 4(3), e4786. https://doi.org/10.1371/journal.pone.0004786

Beran, M. J. (2004). Long-term retention of the differential values of Arabic numerals by chimpanzees (Pan troglodytes). Animal Cognition, 7(2), 86-92. https://doi.org/10.1007/s10071-003-0191-X

Beran, M. J., Harris, E. H., Evans, T. A., Klein, E. D., Chan, B., Flemming, T. M., \& Washburn, D. A. (2008). Ordinal judgments of symbolic stimuli by capuchin monkeys (Cebus apella) and rhesus monkeys (Macaca mulatta): The effects of differential and nondifferential reward. Journal of Comparative Psychology, 122(1), 52-61. 
https://doi.org/10.1037/0735-7036.122.1.52

Bialystok, E., \& Codd, J. (2000). Representing quantity beyond whole numbers: Some, none, and part. Canadian Journal of Experimental Psychology, 54(2), 117-128. https://doi.org/10.1037/h0087334

Biro, D., \& Matsuzawa, T. (2001). Use of numerical symbols by the chimpanzee (Pan troglodytes): Cardinals, ordinals, and the introduction of zero. Animal Cognition, 4(3-4), 193-199. https://doi.org/10.1007/s100710100086

Bortot, M., Agrillo, C., Avarguès-Weber, A., Bisazza, A., Miletto Petrazzini, M. E., \& Giurfa, M. (2019). Honeybees use absolute rather than relative numerosity in number discrimination. Biology Letters, 15, 20190138. https://doi.org/10.1098/rsbl.2019.0138

Boysen, S. T., \& Berntson, G. G. (1989). Numerical competence in a chimpanzee (Pan troglodytes). Journal of Comparative Psychology, 103(1), 23-31. https://doi.org/10.1037/0735-7036.103.1.23

Brannon, E. M., \& Terrace, H. S. (1998). Ordering of the numerosities 1 to 9 by monkeys. Science, 282, 746-749. https://doi.org/10.1126/science.282.5389.746

Brannon, E. M., \& Terrace, H. S. (2000). Representation of the numerosities 1-9 by rhesus macaques (Macaca mulatta). Journal of Experimental Psychology: Animal Behavior Processes, 26(1), 31-49. https://doi.org/10.1037/0097-7403.26.1.31

Brannon, E. M., Wusthoff, C. J., Gallistel, C. R., \& Gibbon, J. (2001). Numerical subtraction in the pigeon: Evidence for a linear subjective number scale. Psychological Science, 12(3), 238-243. https://doi.org/10.1111/1467-9280.00342

Brysbaert, M. (1995). Arabic number reading: On the nature of the numerical scale and the origin of phonological recording. Journal of Experimental Psychology: General, 124(4), 434-452. https://doi.org/10.3758/BF03207272

Cammaerts, M. C., \& Cammaerts, D. (2014). Comparative outlook over three Myrmica species' biotopes and foragers' know-how, Biologia, 69, 1051-1058. https://doi.org/10.2478/s11756-014-0399-z

Cammaerts, M. C., \& Cammaerts, R. (2019a). Ants are at the first stage of the notion of zero. International Journal of Biology, 11(1), 54-65 https://doi.org/10.5539/ijb.v11n1p54

Cammaerts, M. C., \& Cammaerts, R. (2019b). Ants' notion of zero through the perception of the absence of an odor. International Journal of Biology, 11(2), 1-12. https://doi.org/10.5539/ijb.v11n2px

Cammaerts, M. C., \& Cammaerts, R. (2019c). Ants' capability of adding numbers of identical elements. International Journal of Biology, 11(3), 25-36. https://doi.org/10.5539/ijb.v11n3p25

Cammaerts, M. C., \& Cammaerts, R. (2019d). Ants fail to add numbers of same elements seen consecutively. International Journal of Biology, 11(3), 37-48. 
https://doi.org/10.5539/ijb.v11n3p37

Cammaerts, M. C., \& Cammaerts, R. (2019e). Ants correctly locate the zero in a continuous series of numbers. International Journal of Biology, 11(4), 16-25. https://doi.org/10.5539/ijb.v11n4p16

Cammaerts, M. C., \& Cammaerts, R. (2019f). Subtraction-like effect in an ant faced with numbers of elements including a crossed one. International Journal of Biology, 11(4), 51-66. https://doi.org/10.5539/ijb.v11n4p51

Cammaerts, M. C., \& Cammaerts, R. (2019g). Left to right oriented number scaling in an ant. International Journal of Biology, 11(4), 67-79. https://doi.org/10.5539/ijb.v11n4p67

Cammaerts, M. C., \& Cammaerts, R. (2020a). Ants' capability of adding and subtracting odors. International Journal of Biology, 12(1), 1-13. https://doi.org/10.5539/ijb.v12n1p1

Cammaerts M. C., \& Cammaerts, R. (2020b). Ants'numerosity ability defined in nine studies. Journal of Biology and Life Sciences, 11(1), 121-142. https://doi.org/10.5296/jbls.v11i1.16278

Cammaerts M. C., \& Cammaerts R. (2020c). Young ants already possess a number line. International Journal of Biology, 12(2), 1-12. https://doi.org/10.5539/ijb.v12n2p1

Cammaerts M. C., \& Cammaerts, R. (2020d). Ants acquire the notion of zero through experiences. International Journal of Biology, 12(2), 13-25. https://doi.org/10.5539/ijb.v12n2p13

Cammaerts M. C., \& Cammaerts R. (2020e). Influence of shape, color, size and relative position of elements on their counting by an ant. International Journal of Biology, 12(2), 26-40. https://doi.org/10.5539/ijb.v12n2p26

Cammaerts M. C., \& Cammaerts R. (2020f). Ants can associate a symbol with a number of elements through conditioning. International Journal of Biology, 12(3), 1-13. https://doi.org/10.5539/ijb.v12n3p1

Cammaerts M. C., \& Cammaerts R. (2020g). Ants can acquire multiple symbolisms. International Journal of Biology, 12(3), 18-26. https://doi.org/10.5539/ijb.v12n3p18

Cammaerts M. C., \& Cammaerts R. (2020h). Ants can add up using learned numeric symbols. International Journal of Biology, 12(3), 27-39. https://doi.org/10.5539/ijb.v12n3p27

Cammaerts M. C., \& Cammaerts R. (2020i). Ants' acquisition of a symbol for zero and its use to add. International Journal of Biology. In press.

Cammaerts, R., \& Cammaerts, M. C. (2020) Ants' mental positioning of amounts on a number line. International Journal of Biology, 12(1), 30-45. https://doi.org/10.5539/ijb.v12n1p30

Cantlon, J. F., \& Brannon, E. M. (2006). Shared system for ordering small and large numbers in monkeys and humans. Psychological Science, 17(5), 401-406. 
https://doi.org/10.1111/j.1467-9280.2006.01719.x

De Hevia, M. D., \& Spelke, E. S. (2010). Number-space mapping in human infants. Psychological Science, 21(5), 653-660. https://doi.org/10.1177/0956797610366091

De Hevia, M. D., Girelli, L, Addabbo, M., \& Cassia, V. M. (2014a). Human infants' preference for left-to-right oriented increasing numerical sequences. PLoS ONE, 9(5), e96412. https://doi.org/10.1371/journal.pone.0096412

De Hevia, M. D., Izard, V., Coubart, A., Spelke, E. S., \& Streri, A. (2014b). Representations of space, time, and number in neonates. PNAS, 111(13), 4809-4813. https://doi.org/10.1073/pnas.1323628111

De Hevia, M. D., Vanderslice, M., \& Spelke, E. S. (2012). Cross-dimensional mapping of number, length and brightness by preschool children. PloS ONE, 7(4), e35530. https://doi.org/10.1371/journal.pone.0035530

Dehaene, S. (2011). The number sense: how the mind creates mathematics. USA, Oxford University Press. ISBN 978-0-19-975387.1

Dehaene, S., Izard, V., Spelke, E., \& Pica, P. (2008). Log or linear? Distinct intuitions of the number scale in Western and Amazonian cultures. Science, 320, 1217-1220. https://doi.org/10.1126/science. 1156540

Ditz, H. M., \& Nieder, A. (2016). Numerosity representations in crows obey the Weber-Fechner law. Proceedings of the Royal Society, B, 283, 20160083. https://doi.org/10.1098/rspb.2016.0083

Emmerton, J., \& Renner, J. C. (2006). Scalar effects in the visual discrimination of numerosity by pigeons. Learning \& Behavior, 34(2), 176-192. https://doi.org/10.3758/BF03193193

Flombaum, J. I., Junge, J. A., \& Hauser, M. D. (2005). Rhesus monkeys (Macaca mulatta) spontaneously compute addition operations over large numbers. Cognition, 97, 315-325. https://doi.org/10.1016/j.cognition.2004.09.004

Gallistel, C. R., Brannon, E. M., Gibbon, J., \& Wusthoff, C. J. (2001). Response to Dehaene. Psychological Science, 12(3), 247. https://doi.org/10.1111/1467-9280.00344

Garland, A., \& Low, J. (2014). Addition and subtraction in wild New Zealand robins. Behavioural Processes, 109, 103-110. https://doi.org/10.1016/j.beproc.2014.08.022

Gazes, R. P., Diamond, R. F. L., Hope, J. M., Caillaud, D., Stoinski, T. S., \& Hampton, R. R. (2017). Spacial representation of magnitude in gorillas and orangutans. Cognition, 168, 312-319. https://doi.org/10.1016/j.cognition.2017.07.010

Gross, H. J., Pahl, M., Si, A., Zhu, H., Tautz, J., \& Zhang, S. (2009). Number-based visual generalisation in the honeybee. PLoS ONE, 4(1), e4263. https://doi.org/10.1371/journal.pone.0004263 
Hauser, M. D., Carey, S., \& Hauser, L. B. (2000). Spontaneous number representation in semi-free-ranging rhesus monkeys. Proceedings of the Royal Society of London B: Biological Sciences, 26(1445), 829-833. https://doi.org/10.1098/rspb.2000.1078

Hauser, M. D., MacNeilage, P., \& Ware, N. (1996). Numerical representations in primates. Proceedings of the National Academy of Sciences (USA), 93, 1514-1517. https://doi.org/10.1073/pnas.93.4.1514

Howard, S. R., Avarguès-Weber, A., Garcia, J. E., Greentree, A. D., \& Dyer, A. G. (2018). Numerical ordering of zero in honey bees. Science, 360(6393), 1124-1126. https://doi.org/10.1126/science.aar4975

Howard, S. R., Avarguès-Weber, A., Garcia, J. E., Greentree, A. D., \& Dyer, A. G. (2019a). Numerical cognition in honeybees enables addition and subtraction. Cognitive Neuroscience, 5, 1-6. https://doi.org/10.1126/sciadv.aav0961

Howard S. R., Avarguès-Weber, A., Garcia, J. E., Greentree, A. D., \& Dyer, A. G. (2019b). Achieving arithmetic learning in honeybees and examining how individuals learn. Communicative \& Integrative Biology, 12(1), 166-170. https://doi.org/10.1080/19420889.2019.1678452

Howard, S. R., Avarguès-Weber, A., Garcia, J. E., Greentree, A. D., \& Dyer, A. G. (2019c). Symbolic representation of numerosity by honeybees (Apis mellifera): matching characters to small quantities. Proceedings of the Royal Society B, 286, 20190238. https://doi.org/10.1098/rspb.2019.0238

Irie, N., Hiraiwa-Hasegawa, M., \& Kutsukake, N. (2019). Unique numerical competence of Asian elephants on the relative numerosity judgment task. Journal of Ethology, 37, 111-115. https://doi.org/10.1007/s10164-018-0563-y

Jordan, K. E., \& Brannon, E. M. (2006). Weber's law influences numerical representations in rhesus macaques (Macaca mulatta). Animal Cognition, 9, 159-172. https://doi.org/10.1007/s10071-006-0017-8

Kucian, K., Grond, U., Rotzer, S., Henzi, B., Schonmann, C., Plangger, F., ... von Aster, M. (2011). Mental number line training in children with developmental dyscalculia. NeuroImage, 57(3), 782-795. https://doi.org/10.1016/j.neuroImage.2011.01.070

Laski, E. V., \& Siegler, R. S. (2007). Is 27 a big number? Correlational and causal connections among numerical categorization, number line estimation, and numerical magnitude comparison. Child Development, 78(6), 1723-1743. https://doi.org/10.1111/j.1467-8624.2007.01087.x

Matsuzawa, T. (1985). Use of numbers by a chimpanzee. Nature, 315(2), 57-59. https://doi.org/10.1038/315057a0

Mazzocco, M. M., Feigenson, L., \& Halberda, J. (2011). Preschoolers' precision of the approximate number system predicts later school mathematics performance. PLoS One, 6, e23749. https://doi.org/10.1371/journal.pone.0023749 
Merrit, D. J., Rugani, R., \& Brannon, E. M. (2009). Empty sets as part of the numerical continuum: Conceptual precursors to the zero concept in rhesus monkeys. Journal of Experimental Psychology: General, 138, 258-269. https://doi.org/10.1037/a0015231

Merritt, D. J., \& Brannon, E. M. (2013). Nothing to it: precursors to a zero concept in preschoolers. Behavioural Processes, 93, 91-97. https://doi.org/10.1016/j.beproc.2012.11.001

Merten, K., \& Nieder, A. (2008). Compressed scaling of abstract numerosity representations in adult humans and monkeys. Journal of Cognitive Neuroscience, 21(2), 333-346. https://doi.org/10.1162/jocn.2008.21032

Murofushi, K. (1997). Numerical matching behavior by a chimpanzee (Pan troglodytes): Subitizing and analogue magnitude estimation. Japanese Psychological Research, 39(32), 140-153. https://doi.org/10.1111/1468-5884.00050

Nieder, A. (2009). Prefrontal cortex and the evolution of symbolic reference. Current Opinion in Neurobiology, 19(1), 99-108. https://doi.org/10.1016/j.conb.2009.04.008

Nieder, A. (2016). Representing something out of nothing: The drawing of zero. Trends in Cognitive Sciences, 20(11), 830-842. https://doi.org/10.1016/j.tics.2016.08.008

Nieder, A., \& Miller, E. K. (2003). Coding of cognitive magnitude: Compressed scaling of numerical information in the primate prefrontal cortex. Neuron, 37, 149-157. https://doi.org/10.1016/S0896-6273(02)01144-3

Nieder, A., Freedman, D. J., \& Miller, E. K. (2002). Representation of the quantity of visual items in the primate prefrontal cortex. Science, 297, 1708-1711. https://doi.org/10.1126/science.1072493

Okuyama, S., Kuki, T., \& Mushiake, H. (2015). Representation of the numerosity 'zero' in the parietal cortex of the monkey. Scientific Reports, 5, 10059. https://doi.org/10.1038/srep10059

Olthof, A., \& Roberts W. A. (2000). Summation of symbols by pigeons (Columba livia): the importance of number and mass of reward items. Journal of Comparative Psychology, 111(2), 158-166. https://doi.org/10.1037/0735-7036.114.2.158

Olthof, A., Iden, C. M., \& Roberts, W. A. (1997). Judgments of ordinality and summation of number symbols by squirrel monkeys (Saimiri sciureus). Animal Behavior Processes, 23(3), 325-339. https://doi.org/10.1037/0097-7403.23.3.325

Pepperberg, I. M. (1988). Comprehension of "absence" by an African grey parrot: Learning with respect to questions of same/different. Journal of the Experimental Analysis of Behavior, 50, 553-564. https://doi.org/10.1901/jeab.1988.50-553

Pepperberg, I. M. (2006a). Grey parrot (Psittacus erithacus) numerical abilities: Addition and further experiments on a zero-like concept. Journal of Comparative Psychology, 120(1), 1-11. https://doi.org/10.1037/0735-7036.120.1.1

Pepperberg, I. M. (2006b). Ordinality and inferential abilities of a grey parrot (Psittacus 
erithacus). Journal of Comparative Psychology, 120(3), 205-216. https://doi.org/10.1037/0735-7036.120.3.205

Pepperberg, I. M. (2012). Further evidence for addition and numerical competence by a grey parrot (Psittacus erithacus). Animal Cognition, 15(4), 711-717. https://doi.org/10.1007/s10071-012-0470-5

Pepperberg, I. M., \& Gordon, J. D. (2005). Number comprehension by a grey parrot (Psittacus erithacus), including a zero-like concept. Journal of Comparative Psychology, 119, 197-209. https://doi.org/10.1037/0735-7036.119.2.197

Pinhas, M., \& Tzelgov, J. (2012). Expanding in the mental number line: Zero is perceived as the "smallest". Journal of Experimental Psychology: learning, memory, and cognition, 38(5), 1187-1205. https://doi.org/10.1037/a0027390

Pixner, S., Dresen, V., \& Moeller, K. (2018). Differential development of children's understanding of the cardinality of small numbers and zero. Frontiers in Psychology, 9, 1636. https://doi.org/10.3389/fpsyg.2018.01636

Range, F., Jenikejew, J., Schröder, I., \& Virányi, Z. (2014). Difference in quantity discrimination in dogs and wolves. Frontiers in Psychology. 5, 1299. https://doi.org/10.3389/fpsyg.2014.01299

Rodríguez, R. L., Briceño, R. D., Briceño-Aguilar, E., \& Höbel, G. (2015). Nephila clavipes spiders (Araneae: Nephilidae) keep track of captures prey counts: testing for a sense of numerosity in an orb-weaver. Animal Cognition, 18(1), 307-314. https://doi.org/10.1007/s10071-014-0801-9

Rugani, R, Fontanari, L., Simoni, E., Regolin, L., \& Vallortigara, G. (2009). Arithmetic in newborn chicks. Proceedings of the Royal Society, B, 276, 2451-2460. https://doi.org/10.1098/rspb.2009.0044

Rugani, R., \& de Hevia, M. D. (2017). Number-space associations without language: evidence from preverbal human infants and non-human animal species. Psychonomic Bulletin \& Review, 24(2), 352-369. https://doi.org/10.3758/s13423-016-1126-2

Rugani, R., Rosa-Salva, O., \& Regolin, L. (2014). Lateralized mechanisms for encoding of object. Behavioral evidence from an animal model: the domestic chick (Gallus gallus). Frontiers in Psychology, 5(150), 1-8. https://doi.org/10.3389/fpsyg.2014.00150

Rugani, R., Vallortigara, G., Priftis, K, \& Regolin, L. (2015). Number-space mapping in the newborn chick resembles humans' mental number line. Science, 347(6221), 534-536.https://doi.org/10.1126/science.aaa1379

Rumbaugh, D. M., Savage-Rumbauch, S., \& Hegel, M. T. (1987). Summation in the chimpanzee (Pan troglodytes). Journal of Experimental Psychology: Animal and Behaviour Processes, 13(2), 107-115. PMID: 3572305. https://doi.org/10.1037/0097-7403.13.2.107

Siegel, S., \& Castellan, N. J. (1988). Nonparametric statistics for the behavioural sciences. 
Singapore,

McGraw-Hill

Book

Company.

https://www.amazon.com/Sidney-Siegel...Statistics.../B008WDIR6

Siegler, R. S., Thompson, C. A., \& Opfer, J. E. (2009). The logarithmic-to-linear shift: One learning sequence, many tasks, many time scales. Mind Brain Education, 3, 143-150. https://doi.org/10.1037/a0014239

Tomonaga, M. (2008). Relative numerosity discrimination by chimpanzees (Pan troglodytes): evidence for approximate numerical representations. Animal Cognition. 11, 43-57. https://doi.org/10.1007/s10071-007-0089-0

Washburn, D. A., \& Rumbauch, D. M. (1991). Ordinal judgments of numerical symbols by macaques (Macaca mulatta). Psychological Science, 2(3), 190-193. https://doi.org/10.1111/j.1467-9280.1991.tb00130.x

Wellman, H. M., \& Miller, K. F. (1986). Thinking about nothing: Development of concepts of zero. British Journal of Developmental Psychology, 4, 31-42. https://doi.org/10.1111/j.2044-835X.1986.tb00995.x

West, R. E., \& Young, R. J. (2002). Do domestic dogs show any evidence of being able to count? Animal Cognition, 5, 183-186. https://doi.org/10.1007/s10071-002-0140-0

Wheeler, M. M., \& Feghali, I. (1983). Much ado about nothing: Preservice elementary school teachers' concept of zero. Journal of Research in Mathematics Education, 14(3), 147-155. https://doi.org/10.2307/748378

Xia, L., Siemann, M., \& Delius J. D. (2000). Matching of numerical symbols with number of responses by pigeons. Animal Cognition, 3, 35-43. https://doi.org/10.1007/s100710050048

Xu, F., \& Spelke, E. S. (2000). Large number discrimination in 6-month-old infants. Cognition, 74, B1-B11. https://doi.org/10.1016/S0010-0277(99)00066-9

Zorzi, M., Priftis, K., \& Umiltà, C. (2002). Brain damage: neglect disrupts the mental number line. Nature, 417, 138-139. https://doi.org/10.1038/417138a

\section{Copyright Disclaimer}

Copyright for this article is retained by the author(s), with first publication rights granted to the journal.

This is an open-access article distributed under the terms and conditions of the Creative Commons Attribution license (http://creativecommons.org/licenses/by/4.0/). 